\title{
The A10389G polymorphism of $N D 3$ gene and breast cancer: A meta-analysis
}

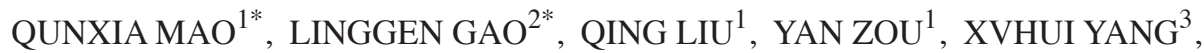 \\ $\mathrm{HONGWEI} \mathrm{WANG}^{4}$, QIAN WANG ${ }^{5}$ and $\mathrm{HUA} \mathrm{YU}^{1}$ \\ ${ }^{1}$ National Research Institute for Family Planning; ${ }^{2}$ Department of Geriatric Cardiology, General Hospital of Chinese \\ People's Liberation Army, Beijing; ${ }^{3}$ Hangzhou Centre for Disease Control and Prevention, Zhejiang; \\ ${ }^{4}$ Department of Safety Engineering, China Institute of Industrial Relations; ${ }^{5}$ Cardiovascular Institute and Fu Wai Hospital, \\ Chinese Academy of Medical Sciences and Peking Union Medical College; Beijing, P.R. China
}

Received October 12, 2012; Accepted January 2, 2013

DOI: $10.3892 /$ br. 2013.58

\begin{abstract}
Previous studies have reported the association between A10389G polymorphism of the dehydrogenase subunit 3 (ND3) gene and breast cancer risk with conflicting results. To explore this association, we conducted a meta-analysis on 5,580 patients and 5,749 controls from eligible published studies. Six reports (11 study populations) were included in this meta-analysis. Compared with the individuals with the $\mathrm{G}$ allele, individuals carrying the A allele did not exhibit increased breast cancer risk. The odds ratio (OR) and 95\% confidence interval (CI) were 1.02 and 0.79-1.31, respectively. Stratified analyses were carried out according to ethnicity and source of controls. The corresponding ORs (95\% CIs) were 1.20 (0.90-1.86) for African-American, 0.47 (0.03-7.64) for European and $0.89(0.70-1.14)$ for mixed populations, respectively. A single study on Asian populations yielded an OR $(95 \% \mathrm{CI})$ of $0.56(0.32-1.00)$. The corresponding ORs (95\% CIs) were $1.12(0.23-5.47)$ for hospital-based studies, and $0.98(0.76-1.27)$ for population-based studies. Only one study did not mention the source of control, the OR $(95 \% \mathrm{CI})$ of which was 1.80 (1.15-2.82). Results of the present study suggested that the ND3 gene A10389G polymorphism may not be an independent risk factor for breast cancer. However, additional studies should be performed to clarify the possible roles of the ND3 A10398G polymorphism in the etiology of breast cancer.
\end{abstract}

Correspondence to: Dr Qunxia Mao, National Research Institute for Family Planning, 12 Dahuisi Rd., Haidian District, Beijing 100081, P.R. China

E-mail: flysolojingyamao@gmail.com

*Contributed equally

Key words: A10389G polymorphism, dehydrogenase subunit 3 gene, breast cancer, meta-analysis

\section{Introduction}

Breast cancer is known to have a complex, multi-factorial etiology, with contributions from genetic and environmental factors (1-2). Certain low-penetrance genes have been reported to be involved in breast cancer carcinogenesis (3).

Mitochondria are the major source of Reactive Oxygen Species (ROS) (4), and are important in cell energy production (5), by playing active roles in cell death and cell proliferation (6). Mitochondria may act individually or in combination with other mitochondrial DNA variations or through interaction with nuclear genes or environmental factors to modify cancer risk (7-9). The 10398 nucleotide position (np) in the human mitochondrial genome is highly polymorphic (3). A10398G polymorphism (A to $G$ transition) results in an amino acid change from threonine to alanine. Additionally, the A10398G polymorphism in the nicotinamide adenine dinucleotide (NADH) dehydrogenase subunit 3 (ND3) gene was shown to modify the risk of certain aging-associated diseases (10-13).

The local ROS produced by redox cycling metabolites within breast cells with an increase in cellular oxidative stress was suggested to lead to carcinogenesis (4). The association between ND3 A10389G polymorphism and breast cancer risk has been previously investigated $(3,5,7)$, however, the results obtained in this study were not always consistent. A meta-analysis on eligible case-control studies was conducted herein to estimate the association between A10389G polymorphism and the risk of breast cancer.

\section{Materials and methods}

Search strategy. Searches were conducted using PubMed, Embase and the Cochrane Library in English and VIP, CNKI, and Sinomed (up to November 15, 2011) in Chinese. The key words and subject terms used were: 'ND3' or 'mitochondrial A10389G polymorphism' and 'breast cancer'. We also used the search terms: 'ND3' and 'breast cancer' and 'genetic association' in HuGE Navigator. References of received articles were also searched. Articles included in this meta-analysis were defined as: i) case-control study (including nested case-control study); ii) not family-based study; iii) evaluation of the relation- 
Table I. Characteristics of studies included in this analysis.

\begin{tabular}{|c|c|c|c|c|c|c|c|c|c|c|}
\hline \multirow[b]{3}{*}{ ID } & \multirow{3}{*}{$\begin{array}{l}\text { First } \\
\text { author }\end{array}$} & \multirow{3}{*}{$\begin{array}{c}\text { Year } \\
\text { (study) }\end{array}$} & \multirow[b]{3}{*}{ Country } & \multirow[b]{3}{*}{ Ethnicity } & \multirow{3}{*}{$\begin{array}{c}\text { Genotyping } \\
\text { method }\end{array}$} & \multirow{3}{*}{$\begin{array}{l}\text { Source of } \\
\text { controls }\end{array}$} & \multirow{3}{*}{$\begin{array}{l}\text { Sample size } \\
\text { (case/control) }\end{array}$} & \multirow{2}{*}{\multicolumn{2}{|c|}{$\frac{\text { Allele distribution }}{\text { (case/control) }}$}} & \multirow[b]{3}{*}{ Refs. } \\
\hline & & & & & & & & & & \\
\hline & & & & & & & & A & G & \\
\hline 1 & Fang & 2010 & China & Asian & Sequence & $\mathrm{HB}$ & $104 / 114$ & $28 / 45$ & $76 / 69$ & 16 \\
\hline \multirow[t]{2}{*}{2} & \multirow[t]{2}{*}{ Pezzotti } & 2009 (1) & USA & Mixed & Taqman & PB & $1561 / 2209$ & $1242 / 2162$ & $319 / 447$ & 17 \\
\hline & & $2009(2)$ & USA & Mixed & Taqman & PB & $678 / 669$ & $529 / 518$ & $149 / 151$ & \\
\hline 3 & Czarnecka & 2009 & Poland & European & PCR and RFLP & PB & $44 / 100$ & $34 / 97$ & $10 / 3$ & 3 \\
\hline \multirow[t]{3}{*}{4} & \multirow[t]{3}{*}{ Setiawan } & 2008 (1) & USA & African-American & Taqman & PB & $541 / 282$ & $38 / 11$ & $493 / 266$ & 18 \\
\hline & & $2008(2)$ & USA & African-American & Taqman & $\mathrm{PB}$ & $391 / 460$ & $26 / 29$ & $391 / 460$ & \\
\hline & & 2008 (3) & USA & African-American & Taqman & $\mathrm{PB}$ & $524 / 236$ & $29 / 16$ & $479 / 219$ & \\
\hline 5 & Bai & 2007 & USA & European & AS-PCR & Not mentioned & $156 / 260$ & $50 / 54$ & $106 / 206$ & 7 \\
\hline \multirow[t]{3}{*}{6} & \multirow[t]{3}{*}{ Canter } & 2005 (1) & USA & African-American & Taqman & HB & $48 / 54$ & $7 / 3$ & $41 / 51$ & 5 \\
\hline & & 2005 (2) & USA & African-American & Taqman & PB & $654 / 605$ & $84 / 52$ & $570 / 553$ & \\
\hline & & $2005(3)$ & USA & European & Taqman & PB & $879 / 760$ & $700 / 601$ & $179 / 159$ & \\
\hline
\end{tabular}

PB, population-based; HB, hospital-based; PCR, polymerase chain reaction; RFLP, restriction fragment length polymorphism; AS-PCR, allele-specific polymerase chain reaction.

Table II. Quality assessment for the eligible studies according to NOS.

\begin{tabular}{|c|c|c|c|c|c|}
\hline ID & First author & Selection (stars) & Comparability (stars) & Exposure (stars) & Refs. \\
\hline 1 & Fang & 3 & 2 & 1 & 16 \\
\hline 2 & Pezzotti & 4 & 2 & 1 & 17 \\
\hline 3 & Czarnecka & 4 & 2 & 1 & 3 \\
\hline 4 & Setiawan & 4 & 2 & 1 & 18 \\
\hline 5 & Bai & 3 & 2 & 1 & 7 \\
\hline 6 & Canter & 3 & 2 & 1 & 5 \\
\hline
\end{tabular}

NOS for case-control study (14): a study can be awarded a maximum of one star for each numbered item within the Selection and Exposure categories. A maximum of four stars can be given for Selection and three stars can be given for Exposure. A maximum of two stars can be given for Comparability. More stars mean higher quality of the article. NOS, Newcastle-Ottawa-Scale.

ship between A10389G polymorphism of the ND3 gene and risk of breast cancer; and iv) utilizing the previous study if multiple studies were based on the same population. We excluded review articles, case reports, editorials and information articles for patients only. Any studies without sufficient information about the ND3 A10389G polymorphism were also excluded.

Data extraction. Two investigators (Q.X. Mao and L.G. Gao) searched the studies, and screened them for inclusion and appraisal. Discrepancies were discussed with all the reviewers. Agreements were reached after discussions. Data including author, year of publication, country, ethnicity, study design, sample size, resources of controls, and the information of the ND3 gene A10398G polymorphism was collected from each publication. The Newcastle-Ottawa-Scale (NOS) was used to quantify study quality (14).

Statistical analysis. Unadjusted odds ratio (OR) with corresponding $95 \%$ confidence interval (CI) of each selected study was first calculated. The pooled OR was examined using the Z-test. Heterogeneity among studies was measured by the Q-statistic and I-square statistic tests. Random-effect models busing DerSimonian and Laird method were used in the meta-analysis. Hardy-Weinberg $(\mathrm{H}-\mathrm{W})$ equilibrium was assessed using Pearson's Chi-square test for the controls in each study.

Stratified analysis was performed according to ethnicity and source of controls. Sensitivity analysis was performed to assess the stability of the results. Potential publication bias was assessed by Begg's funnel plot and Egger's linear regression. Analyses were performed using software Stata, version 8.0. $\mathrm{P}<0.05$ was considered to indicate a statistically significant difference. Tests were two-sided.

\section{Results}

Study characteristics and meta-analysis database. Forty-five potentially related studies were searched based on the search 


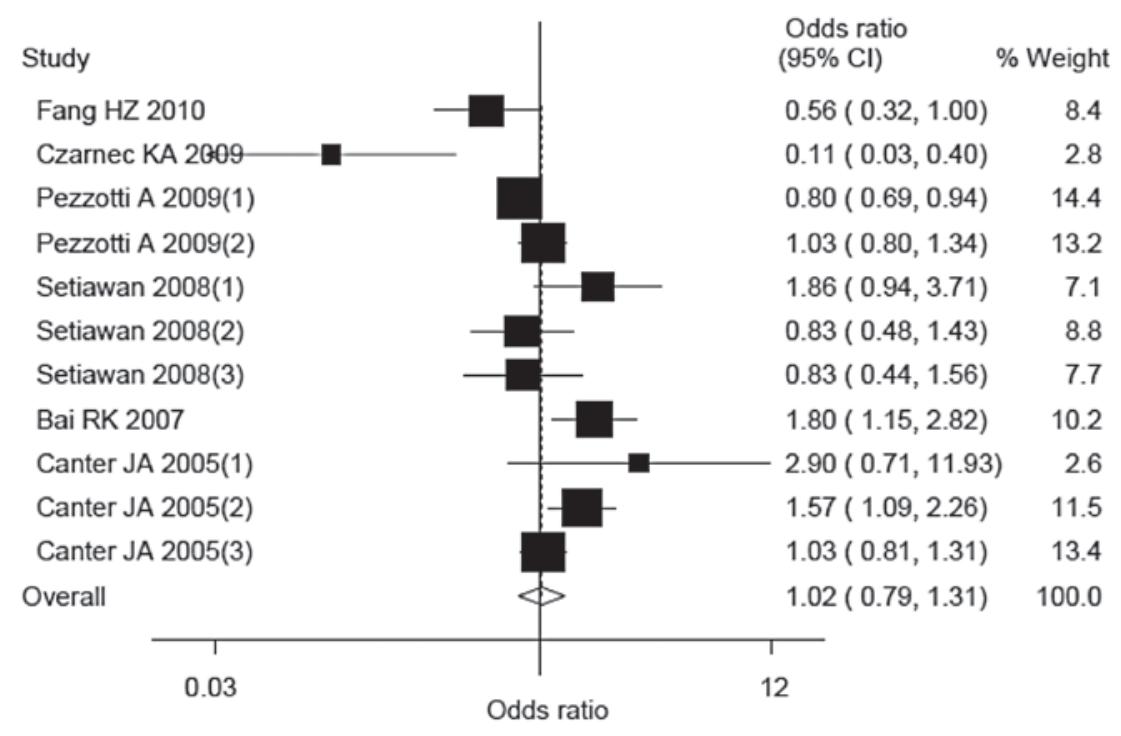

Figure 1. Forest plots of OR with $95 \%$ CI of breast cancer associated with the ND3 gene. A10398G polymorphism was assessed by the random-effects model (A vs. G). Black squares represent the value of OR and the size of the square indicates the inverse proportion relative to its variance. Horizontal line is the $95 \%$ CI of OR. Diamond shows the pooled results. The studies are listed by year of publication. OR, odds ratio; CI, confidence interval.

terms from the databases of PubMed, the HuGE Navigator, Embase and the Cochrane Library in English. No related study in Chinese was identified. One animal-related study, two reviews, and 34 unrelated studies were excluded. Another study concerning the ND3 genotypes was also excluded. Two studies were based on the same population, the second (15) of which was also excluded. Therefore, six studies (3,5,7,16-18) including 11 study populations were included in this meta-analysis, with a total of 5,580 patients and 5,749 controls for the ND3 A10398G polymorphism.

A dataset was established based on the extracted information from each included study (Table I). Cases of breast cancer were confirmed by medical records and clinical examinations. Information on the ND3 A10398G polymorphism was obtained via sequencing, Taqman, allele-specific polymerase chain reaction (PCR) and restriction fragment length polymorphism (RFLP). Quality assessment for the eligible studies according to the NOS is shown in Table II.

Quantitative synthesis. Findings obtained from the eligible studies indicated that compared with individuals carrying the $\mathrm{G}$ allele, individuals carrying the $\mathrm{A}$ allele did not exhibit an increased breast cancer risk. The OR (95\% CI) and $\mathrm{P}_{\text {heterogeneity }}$ value were $1.02(0.79-1.31)$ and $<0.01$, respectively (Fig. 1).

Stratified analyses were performed as per ethnicity and source of controls (Figs. 3 and 4). The corresponding ORs

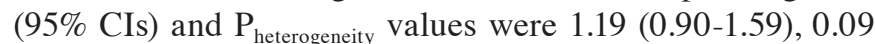
for African-American; 0.47 (0.03-7.64), <0.01 for European; and 0.89 (0.70-1.14), 0.10 for mixed populations, respectively. There was only one Asian study and the OR (95\% CI) was 0.56 (0.32-1.00). The corresponding ORs (95\% CIs) and $\mathrm{P}_{\text {heterogeneity }}$ values were $1.12(0.23-5.47), 0.04$ for hospital-based studies; and $0.98(0.76-1.27),<0.01$ for population-based studies. Only one study did not mention the source of control, with an OR (95\% CI) of $1.80(1.15-2.82)$.

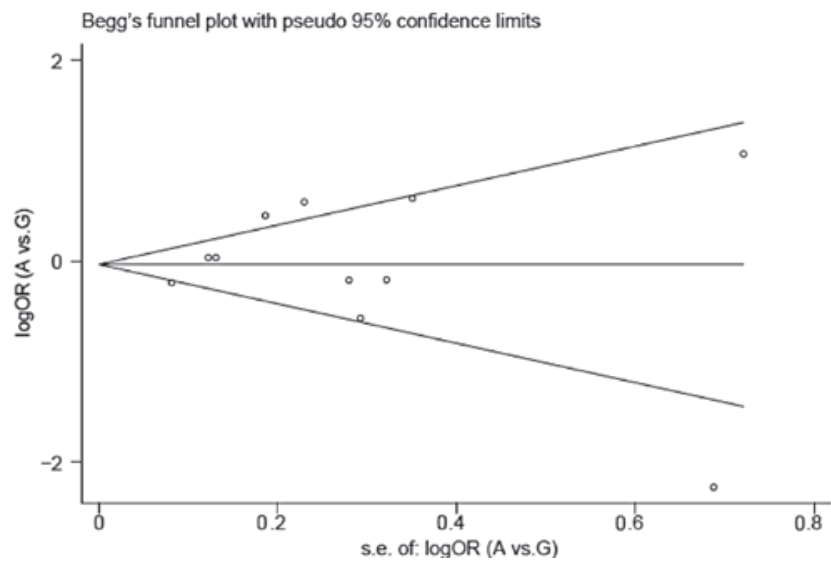

Figure 2. Begg's funnel plot of publication bias test (A vs. G). LogOR represents the nature logarithm of OR. The horizontal line indicates the summary estimate, while the sloping lines are the expected $95 \%$ confidence interval. OR, odds ratio.

Sensitivity analysis. Four study populations [by Czarnecka et al (3), Setiawan et al (one study; 18), Bai et al (7) and Canter et al (one study; 5)] were the main origin of heterogeneity of this meta-analysis. The heterogeneity was decreased following the exclusion of the four studies, with P-values based on the heterogeneity test of 0.02 . The corresponding OR $(95 \% \mathrm{CI})$ were altered to $0.93(0.83-1.03)$, respectively (Fig. 5).

Publication bias. Begg's funnel plots and Egger's tests were conducted to determine publication bias. No publication bias was identified for A10389G polymorphism: P=0.94 (Fig. 2).

\section{Discussion}

This meta-analysis included a total of 5,580 patients and 5,749 controls from six eligible studies (11 study populations). 


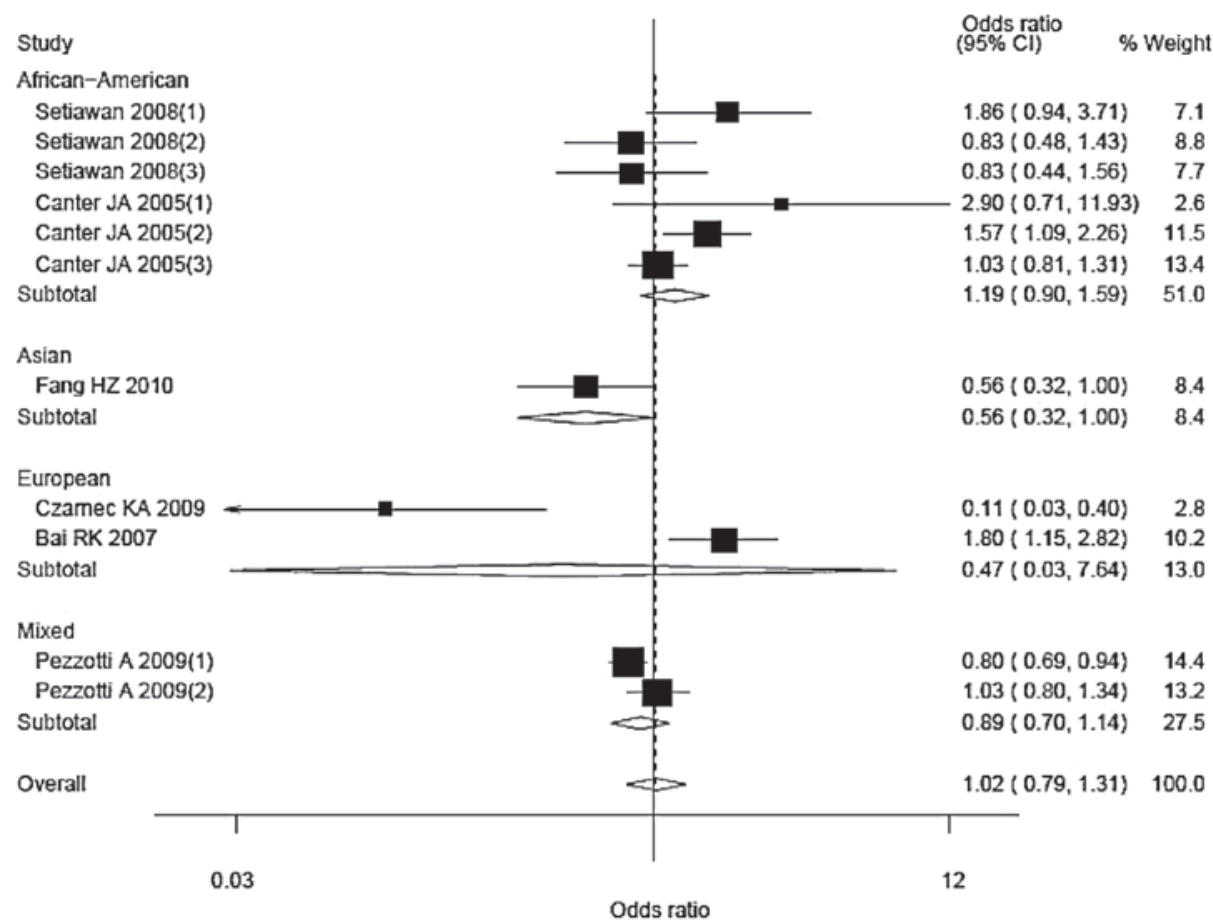

Figure 3. Forest plots of OR with 95\% CI of breast cancer associated with the ND3 gene. A10398G polymorphism according to the ethnicity groups by the random-effects model (A vs. G). Black squares represent the value of OR and the size of the square indicates the inverse proportion relative to its variance. Horizontal line is the $95 \%$ CI of OR. Diamond shows the pooled results. The studies are listed by year of publication. OR, odds ratio; CI, confidence interval.

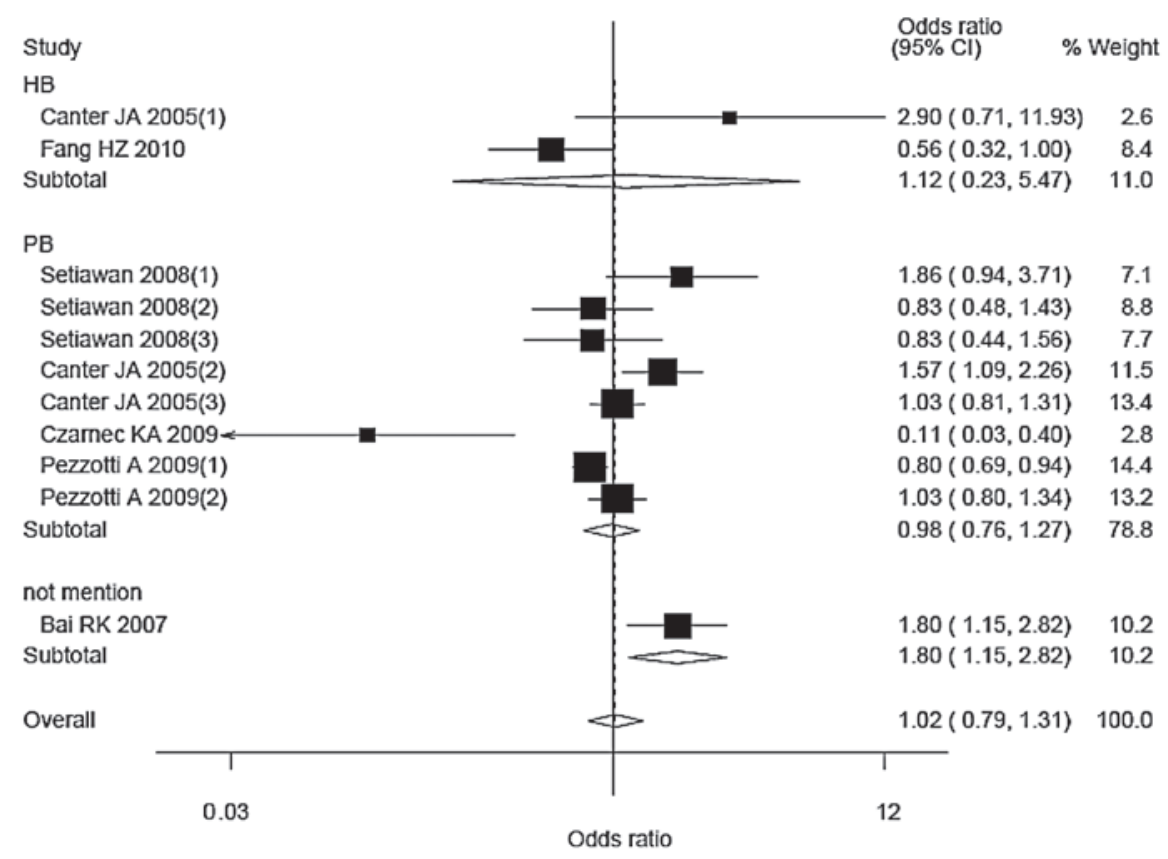

Figure 4. Forest plots of OR with $95 \%$ CI of breast cancer associated with the ND3 gene. A10398G polymorphisms according to different source of controls by the random-effects model (A vs. G). Black squares represent the value of OR and the size of the square indicates the inverse proportion relative to its variance. Horizontal line is the $95 \%$ CI of OR. Diamond shows the pooled results. The studies are listed by year of publication. OR, odds ratio; CI, confidence interval.

The $10398 \mathrm{np}$ is highly polymorphic in the human mitochondrial genome (3). A10398G is probably one of the most thoroughly studied mitochondrial single nucleotide polymorphisms, particularly for its potential effect on tumorigenesis, and it serves as a breast cancer predisposition factor $(16,19)$. In a study based on a regional Chinese population, with a total of 104 cases and 114 controls, $\mathrm{G}$ allele was found to increase the risk of breast cancer, with an OR $(95 \% \mathrm{CI})$ of 1.77 (1.00-3.14) (16). In the study by Bai et al (7), the $10398 \mathrm{G}$ allele was also shown to increase risk of breast cancer in European-American women (7). However, these results were not always consistent. Canter et al (5) detected an 


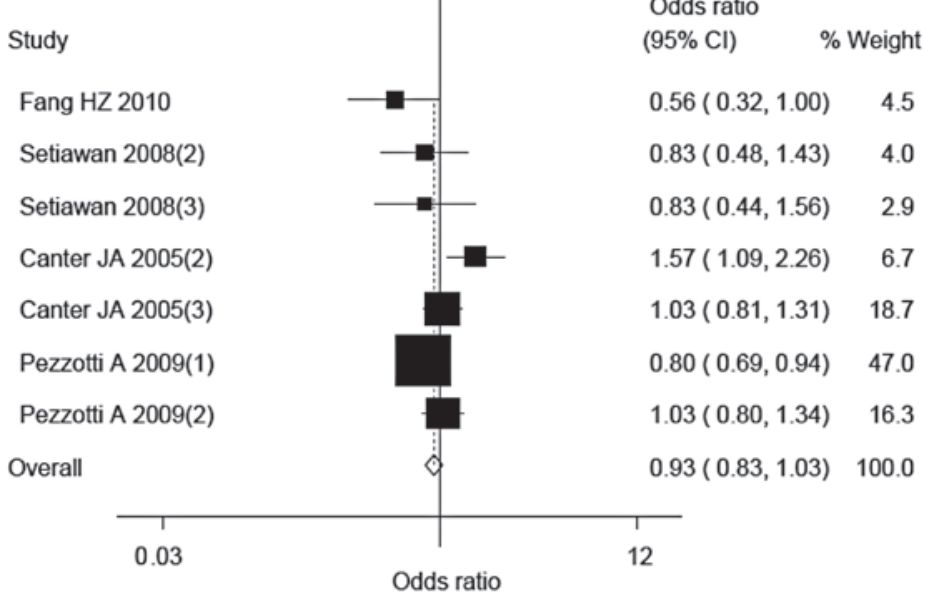

Figure 5. Forest plots of OR with 95\% CI of breast cancer associated with the ND3 gene A10398G polymorphisms by the fixed-effects model for sensitivity analysis (A vs. G). Black squares represent the value of OR and the size of the square indicates the inverse proportion relative to its variance. Horizontal line is the $95 \%$ CI of OR. Diamond shows the pooled results. The studies are listed by year of publication. OR, odds ratio; CI, confidence interval.

inverse association between A10398G polymorphism and the risk of breast cancer (5). African-American women carrying the 10398A allele had a significantly increased risk of breast cancer (OR, 1.60; 95\% CI, 1.10-2.31). However, no statistically significant correlation was identified in Caucasian women. Of note, such a statistically significant association between A10398G polymorphism and the risk of breast cancer was not always duplicated in other studies. Pezzotti et al (17) reported that the frequencies of the $\mathrm{G}$ allele among cases and controls from both the Nurses' Health Study (NHS) population (including 1,561 cases and 2,209 controls) and Women's Health Study (including 678 cases and 669 controls) were similar to each other. Findings of that study also demonstrated that alcohol consumers carrying the $10398 \mathrm{G}$ allele had a higher risk of breast cancer as compared to non-drinkers among the NHS population. However, Setiawan et al (18) observed that G10398A was not significantly associated with breast cancer risk among African-American women. Similarly, no association was detected in three study populations including the San Francisco Bay Area case-control study comprising 541 cases and 282 controls, a nested case-control study based on the Multiethnic Cohort Study comprising 391 cases and 460 controls and a case-control study based on the Los Angeles component of the Women's Contraceptive and Respective Experiences Study and the LIFE study comprising 524 cases and 236 controls. For our meta-analysis, the results also support that individuals carrying A allele did not exhibit an increased risk of breast cancer as per the eligible studies, when compared with individuals carrying the $\mathrm{G}$ allele. Even in stratified analyses, no statistically significant relationship between A10398G genotypes and breast cancer risk was detected.

There are limitations to our meta-analysis that should be considered when interpreting the results. First, since the original data of the reviewed studies were lacking, only unadjusted estimates were assessed and the potential effect on breast cancer risk from other possible variables including age and family history could not be evaluated. At the same time, our evaluation of potential interactions of gene-gene, gene-environ- ment or different polymorphic loci in the same gene that may affect breast cancer risk was limited. Second, the number of studies included in this analysis were not sufficiently large for a comprehensive analysis. Despite the limitations, however, our meta-analysis has important advantages. First, the substantial number of cases and controls included in this meta-analysis significantly increased the statistical power of the analysis as compared to any individual study. Second, the quality of the case-control studies included in the current pooling analysis was satisfactory and met the inclusion criterion.

In conclusion, our meta-analysis shows that ND3 gene A10398G polymorphism might not statistically modulate breast cancer risk. Although a systematic investigation of the relationship between A10398G polymorphisms and the risk of breast cancer could not be conducted because of the aforementioned limitations, it is important to gain a better understanding of the effect of A10398G polymorphisms on breast cancer risk. At the same time, we assume that studies using standardized unbiased methods, enrolling precisely defined breast cancer cases and healthy controls, with more detailed individual data are needed. Furthermore, more and larger studies, particularly studies stratified by gene-gene and gene-environmental interactions, should be performed to clarify the possible roles of the ND3 A10398G polymorphism in the etiology of breast cancer.

\section{Acknowledgements}

The study was supported by the National Nature Science Foundation for Young Scientists of China (grant no. 81102142), the National Research Institute for Family Planning (grant no. 2010GJSSJKA10) and the General Financial Grant from the China Postdoctoral Science Foundation (grant no. 2011M 500154).

\section{References}

1. Olopade OI, Grushko TA, Nanda R and Huo D: Advances in breast cancer: pathways to personalized medicine. Clin Cancer Res 14: 7988-7999, 2008. 
2. Rohan TE, Wong LJ, Wang T, et al: Do alterations in mitochondrial DNA play a role in breast carcinogenesis? J Oncol 2010: 604304, 2010.

3. Czarnecka AM, Krawczyk T, Zdrozny M, et al: Mitochondrial NADH-dehydrogenase subunit 3 (ND3) polymorphism (A10398G) and sporadic breast cancer in Poland. Breast Cancer Res Treat 121: 511-518, 2010.

4. Darvishi K, Sharma S, Bhat AK, et al: Mitochondrial DNA G10398A polymorphism imparts maternal Haplogroup N a risk for breast and esophageal cancer. Cancer Lett 249: 249-255, 2007.

5. Canter JA, Kallianpur AR, Parl FF and Millikan RC: Mitochondrial DNA G10398A polymorphism and invasive breast cancer in African-American women. Cancer Res 65: 8028-8033, 2005.

6. Wallace DC: Mitochondria as chi. Genetics 179: 727-735, 2008.

7. Bai RK, Leal SM, Covarrubias D, et al: Mitochondrial genetic background modifies breast cancer risk. Cancer Res 67: 4687-4694, 2007.

8. Aikhionbare FO, Khan M, Carey D, et al: Is cumulative frequency of mitochondrial DNA variants a biomarker for colorectal tumor progression? Mol Cancer 3: 30, 2004.

9. Datta S, Majumder M, Biswas NK, et al: Increased risk of ora cancer in relation to common Indian mitochondrial polymorphisms and autosomal GSTP1 locus. Cancer 110: 1991-1999, 2007.

10. van der Walt JM, Nicodemus KK, Martin ER, et al: Mitochondrial polymorphisms significantly reduce the risk of Parkinson disease. Am J Hum Genet 72: 804-811, 2003.

11. van der Walt JM, Dementieva YA, Martin ER, et al: Analysis of European mitochondrial haplogroups with Alzheimer disease risk. Neurosci Lett 365: 28-32, 2004.
12. Mancuso M, Conforti FL, Rocchi A, et al: Could mitochondrial haplogroups play a role in sporadic amyotrophic lateral sclerosis? Neurosci Lett 371: 158-162, 2004

13. Giacchetti M, Monticelli A, De Biase I, et al: Mitochondrial DNA haplogroups influence the Friedreich's ataxia phenotype. J Med Genet 41:293-295, 2004.

14. Wells GA, Shea B, O'Connell D, et al: The Newcastle-Ottawa Scale (NOS) for assessing the quality of nonrandomised studies in meta-analyses. Available from: URL: http://www.ohri. $\mathrm{ca} /$ programs/clinical_epidemiology/oxford.asp. Accessed on November 26, 2011.

15. Covarrubias D, Bai RK, Wong LJ and Leal SM: Mitochondrial DNA variant interactions modify breast cancer risk. J Hum Genet 53: 924-928, 2008.

16. Fang H, Shen L, Chen T, et al: Cancer type-specific modulation of mitochondrial haplogroups in breast, colorectal and thyroid cancer. BMC Cancer 10: 421, 2010.

17. Pezzotti A, Kraft P, Hankinson SE, et al: The mitochondrial A10398G polymorphism, interaction with alcohol consumption, and breast cancer risk. PLoS One 4: e5356, 2009.

18. Setiawan VW, Chu LH, John EM, et al: Mitochondrial DNA G10398A variant is not associated with breast cancer in African-American women. Cancer Genet Cytogenet 181: 16-19, 2008.

19. Brandon M, Baldi P and Wallace DC: Mitochondrial mutations in cancer. Oncogene 25: 4647-4662, 2006. 